\title{
THE EVALUATION OF THE BALMER MERGING EFFECTS IN DIFFERENT CHROMOSPHERIC SOLAR FLARE MODELS
}

\author{
A. Falchi ${ }^{1}$, R. Falciani ${ }^{2}$, L.A. Smaldone ${ }^{3}$, G.P. Tozzi ${ }^{1}$ \\ 1 Osservatorio Astrofisico di Areetri, Firenze, Italy \\ 2 Istituto di Astronomia, Universita' di Firenze, Firenze, Italy \\ 3 Dipartim. Sc. Fisiche, Universita' di Napoli, Napoli, Italy
}

\begin{abstract}
Synthetic spectra computed with semiempirical and theoretical models of the flare atmosphere are discussed. Stark profile of the Balmer lines $(n \geq 6)$ and the Batmer continum have been computed according to Donati-Filchi el al. , (1085). A semiempirical model in beiween the F2 and F3 models proposed by Avrett et al. , (1986) might represent a good approximation of the atmosphere of a flare during the impusive phase. Among the theoretical models proposed by Ricchiazzi (1982), the model, indicated as 1073, with very high coronal pressure $\left(P_{0}=1000 \mathrm{dyne}_{\mathrm{cm}} \mathrm{cm}^{-2}\right)$ probably represents the best approximation of the emission observed during the impulsive phase of a cliromospheric flare. Continuum and Balmer lines ought to be measured and interpreted on the same time for a self-consistent diagnostic of the chroniospheric moditication.
\end{abstract}

\section{INTRODUCTION}

The merging of the highly Stark broadened Balmer lines, close to the Balmer continuum limit, has been aiready recognized (Donati- Falchi et al. , 1985) to be an inclicator mainly of the electron density variation in the chromosplere and in the transition region during a flare. Our present interest is to study the influence of the various models of the flare atmospliere on the spectral signatures present in the range between 3600 and $4200 \AA$, i. e. the Balmer lines higher than $I_{6}$ and the Balmer pseudocontinuum near the Balmer discontinuity. Both signatures should be significantly related to the atmospheric modification induced by the flare occurrence.

\section{COMPUTATIONAL METHOD}

The details of the method used to compute the Stark profile of the Balmer lines $(n \geq 6)$ and their merging have been extensively described (Donati- Falchi et al., 1985). In order to compute the absolute continuum emission of a given atmosphere we need to consider the atomic lines "haze" effect, especially important for $\lambda<4000 \AA$. The relative contribution of atomic lines to the total absorption coefficient, as a function of the height in the solar atmosphere, is given for VAL-C model in Vernazza et al., (1976). We assume that the height dependence of the line opacity relative to the total absorption at $\lambda=4000 \AA$ is also valid at the other wavelengths. For the wavelength dependence law we assumed a smoothed fit of the line opacity distribution function given is Vernazza et al. , (1976, Fig. 3). This wavelength dependence is supposed to be the same 


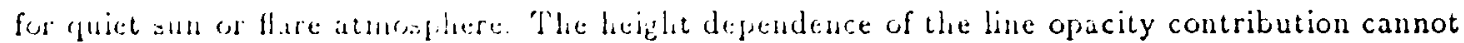
be contidesed the same teccins: of the strong difference in $T_{c}$ and $N_{e}$ distribution between the Hare and gnitet atmers, here. Due to the strong dependence on $N_{e}$ of the line opacity conribution,

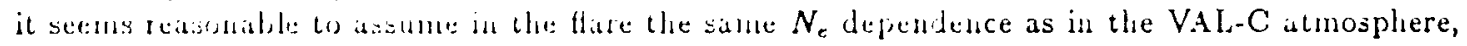
neglecting the Ta deperulence.

The continuras and the Babare lines spectrum, calculated with the above described opacity

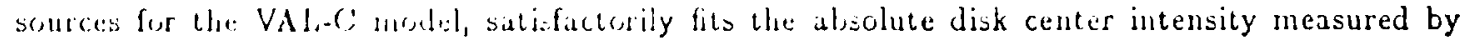
Necke: and lath: ; 1985 ). This, fact makes us confident that our computational procedures are I cli,lste.

\section{HI. SHAMHEMHIRICAL MOHELS}

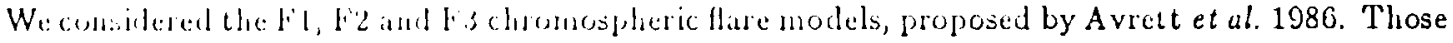
flare mudelis are chan a s orical hy the value of the total pressure $P_{\mathrm{u}}$ in the transition region, which is the bumblary comblitim for the assuned T(m) behaviour. The hychostatic equilibrium conditions deline: the correspunding, vartiatins of the particle density and the geometrical height scale.

T'he lit model is practically invisible in our considered spectral range; only a very small

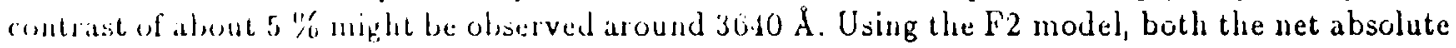
amis. inn in the continumm and (lic slapes of the lines do not match the observed hydrogen spectrum during a llare. As far as the l's model is concerned, we see (Fig. 1) that the general trend of the i)'nthesized spectrum (both the: Balmer psendo-continum and the high Balmer line profiles) fullows the measurements of some flare spectra (Donati-Falchi et al. 1985, Fig. 3 and 4 ) but the alswolute values are ronglily one order of magnitude higher than the observed ones. It seems to us that a semiempirical thare moldel in between $\mathrm{F} 2$ and F3 might represent a good approximation of a phane parallel, homogeneous, hydrostatic model of the chromospheric component of the impulsive phase of a solar flare.

\section{IV. 'THWORFTCAL MODLLS}

Ammg varions existing theoretical models we use the models presented by Ricchiazzi in his thesis (19)82) to compute our synthetic hy drogen spectrum. Ricchiazzi carried out a calculation of the chromospheric respuse 6 both the non-thermal electrons flux with encegy $>20 \mathrm{Kev}$ and the themal comblation. 'The effect of the coronal pressure $P_{0}$ at the apex of the flare loop is also included. We are gratheful to D). R. C. Cunlichl for furnishing the detailed calculation of the

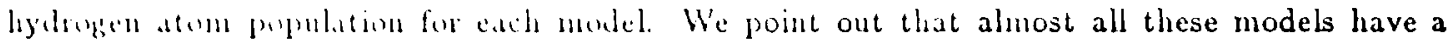
minimum tomperature lower than that of the semiempirical monels and that the height dependence of $T_{c}, N_{r}$ and the ether prameters are strongly affected by the relative importance of the two proposed energey transfer mechanisms and by the value of coronal pressure.

The spectrmm of a thare has been computed with models with constant non-thermal electron thux $b_{\text {an }} 10^{10} e^{10}$ yses ${ }^{-1} \mathrm{~cm}^{-2}$ and with the conductive energy flux $F_{c}$ varying between $10^{6}$

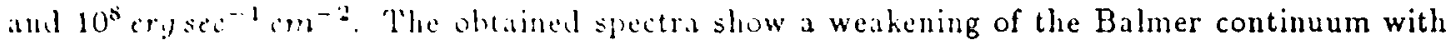
increasing of combuctive thux while the shape of Bahner lines (absorption wings and extremely narrow cunision corel does nut inabe. Coronal pressure changes between 1 and 100 dyne $^{-2}$ do mot atfict the gencral bohision of the computed spectrum. This type of spectrum has never been observal and we may conclude that $F$. is not a dominut energy transfer mechanism during the impulsive phase of a chromosplucrie thare, when the Balmer emission spectrum is well detectable.

The Hux of the monthermaledecions is the main heating source in the chromosphere below 
the TR and only for high coronal pressure becomes important in the TR too. Flare spectra have been computed for models with $F_{c}=10^{7} \mathrm{ergsec}-1 \mathrm{~cm}^{-2}$ and $F_{20}$ vilues varying between 0 and $10^{11} \mathrm{erg} \mathrm{sec}^{-1} \mathrm{cin}^{-2}$. We puint out that a relative increase of the $F_{20}$ importance produces an increase of the Balmer pseudo-continum emission, with minor effects on the Balmer lines intensities and profiles for coronal pressure $1 \leq P_{0} \leq 100$ dyne $\mathrm{cm}^{-2}$. High value of $P_{0}=1000$ dyne $\mathrm{cm}^{-2}$ makes important the heating by non-thermal electrons in the TR too. The net emission of a flare computed for models 1070 and 1073 are shown in Fig. 2-a and -b. We believe that no flare spectra have been observed with slape of Fig. 2-a, while the general shape of the spectrum and the absolute value of the Balmer pseudo-continum and emission lines of Fig 2-b tit reasonably the measurements of these quantities as quoted in the literature. The model 1073 probably represents the best approximation of the atmosphere of a flare during the impulsive phase. This means that the pseudo-continum and the Balmer lines higher thin $I I_{0}$ represent an efficient spectral signature of the relative importance of $F_{20}$ relative to $F_{c}^{\prime}$.

\section{CONCLUSIONS}

Taking into consideration the possible relationships between the signatures in this spectral range and the values of physical parameters as $N_{c}$ and $T_{c}$ of the various considered models we can conclude that:

- the total intensity and the wing profiles of the high Balmer lines $(n \geq 6)$ are sensitive to the values of the maximum electron density above the minimum temperature level;

- the absolute emission of the Balmer pseudo-continuum ( $3600-3700 \AA$ ) is strongly sensitive to the value of the electron column density evaluated above the minimum temperature level where $N_{\epsilon}>10^{13} \mathrm{~cm}^{-3}$; spectrum.

-the minimum temperature value $T_{m i n}$ seems to have a negligible effect on the Balmer

The Balmer pseuclo-continuum, scnsitive to the electron column density evaluated above the minimum temperature level, might be a signature of the importance of non-thermal electron flux in the low chromosphere. The Balmer line profiles, sensitive to the muximum value of $N_{e}$, might be considered a signature of the coronal pressure value. We stress that for a self-consistent diagnostics of the clromospheric modification produced by a flare both continum and Balmer lines must be measured and interpreted. The scparate consideration of only one spectral signature may yield misleading interpretations.

\section{RETERENCES}

Avrett E.H., Machado M.E., Kurucz R.L. 1086, The lower atmosphere of the solar flare, D. Neidig editor, NSO-Sunspot (NM).

Donati-Falclii A., Falciani R., Smaldone L.A. 1085, Astron. Astrophys., 152, 165.

Neckel 11. and Labs D. 1985, Solar Physics, 95, 229

Ricchiazzi P.J. 1982, Ph. D. dissertation, Univerity of California, San Diego.

Vernazza J.E., Avrett E.H., Loeser R. 1976, Ap. J. Suppl. Ser., 90, 1. 


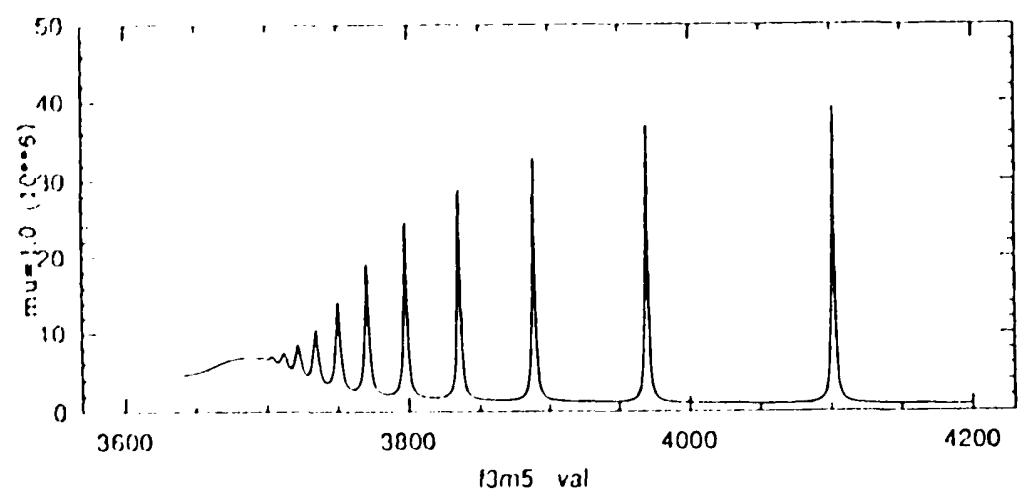

Fipure 1 Jiffucuce $\Delta I(A)$ betwern the alsolute spectral intensity computed for F3 model and the intensily computed for VAL.C atmosplicre at $\mu=1.0$.
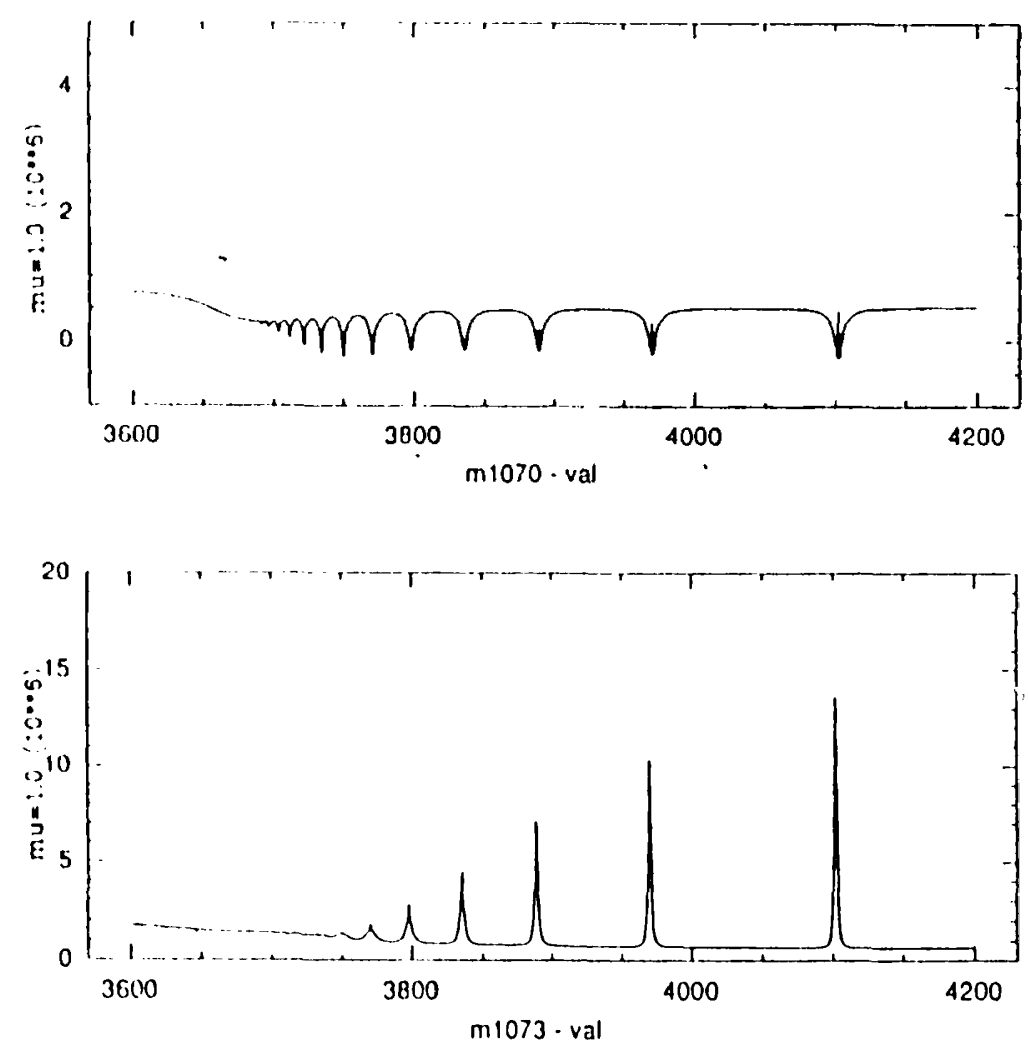

Figure 2 Difference $\Delta I(\lambda)$ hetween the absolute spectral intensity computed for 1070 and 1073 molels and the intensity computed for VA I. C atmosplere at $\mu=1.0$.

a) 1070 molel means : non-lhermal electron nux $=10^{10} \mathrm{erg} \mathrm{sec}^{-1} \mathrm{~cm}^{-2}$; conductive hux =

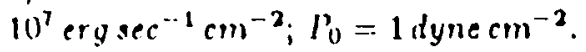

1) 1073 model means : non-thermal electron fux $=10^{10} \mathrm{erg} \mathrm{sec}^{-1} \mathrm{~cm}^{-2}$; conductive flux = $10^{7} \mathrm{ergsec}^{-1} \mathrm{~cm}^{-2} ; \Gamma_{0}=1000$ dyne $\mathrm{cm}^{-2}$. 